\title{
Distributed Space-Time Block Coded Transmission with Imperfect Channel Estimation: Achievable Rate and Power Allocation
}

\author{
Leila Musavian and Sonia Aïssa \\ INRS-EMT, University of Quebec, Montreal, QC, Canada \\ Correspondence should be addressed to Leila Musavian, musavian@emt.inrs.ca
}

Received 2 May 2007; Accepted 27 August 2007

Recommended by R. K. Mallik

This paper investigates the effects of channel estimation error at the receiver on the achievable rate of distributed space-time block coded transmission. We consider that multiple transmitters cooperate to send the signal to the receiver and derive lower and upper bounds on the mutual information of distributed space-time block codes (D-STBCs) when the channel gains and channel estimation error variances pertaining to different transmitter-receiver links are unequal. Then, assessing the gap between these two bounds, we provide a limiting value that upper bounds the latter at any input transmit powers, and also show that the gap is minimum if the receiver can estimate the channels of different transmitters with the same accuracy. We further investigate positioning the receiving node such that the mutual information bounds of D-STBCs and their robustness to the variations of the subchannel gains are maximum, as long as the summation of these gains is constant. Furthermore, we derive the optimum power transmission strategy to achieve the outage capacity lower bound of D-STBCs under arbitrary numbers of transmit and receive antennas, and provide closed-form expressions for this capacity metric. Numerical simulations are conducted to corroborate our analysis and quantify the effects of imperfect channel estimation.

Copyright (C) 2008 L. Musavian and S. Aïssa. This is an open access article distributed under the Creative Commons Attribution License, which permits unrestricted use, distribution, and reproduction in any medium, provided the original work is properly cited.

\section{INTRODUCTION}

An effective way of approaching the promised capacity of multiple-input multiple-output (MIMO) systems is proved to be through space-time coding, which is a powerful technique for achieving both diversity and coding gains over MIMO fading channels [1]. Orthogonal space-time block codes (O-STBCs) that can extract the spatial diversity gains are specially attractive since they drastically simplify maximum likelihood (ML) decoding by decoupling the vector detection problem into simpler scalar detection problems $[2,3]$, thus yielding a process that can be viewed as an orthogonalization of the MIMO channel $[4,5]$.

The use of the MIMO technology along with STBCs is becoming increasingly popular in different wireless systems and networks. Specifically, in sensor and ad hoc networks where nodes are generally limited in terms of the number of antenna elements that can be implemented at the equipment, benefiting from the MIMO technology calls for cooperation between nodes so as to form MIMO antenna arrays in a distributed fashion, and yield the sought for gains of MIMO under space-time block coding.

Recently, there has been increasing interest in distributed space-time coded transmissions which employ STBCs in a cooperative fashion. Indeed, space-time coded cooperative diversity provides an effective way for relaying signals to the end user by multiple disjoint wireless terminals [6]. Cooperative transmit diversity is of particular advantage in sensor networks, where multiple transmit nodes collect information of the same kind and individually transmit the corresponding signals to a given destination, for example, multiple thermal sensors can measure temperature and transmit this information to a device that controls the desired temperature in the space where it operates. These nodes can be deployed to employ distributed STBCs (D-STBCs) in order to cooperatively achieve transmit diversity gains. This is particularly attractive when the links between the transmitting nodes and the receiver (referred to here as 
subchannels) are of different quality, for instance, when a subset of transmitters are required to be positioned at specific locations, for example, sensors measuring the humidity of the soil in a dense environment, wherein not all transmitting nodes can have line-of-sight (LOS) with the receiver.

Performance of D-STBCs with unequal subchannel gains has been investigated in [7] in terms of the outage probability. On the other hand, a memoryless precoder for D-STBCs in MIMO channels with joint transmit-receive correlation is provided in [8]. However, the analyses in $[7,8]$ rely on the availability of perfect state knowledge of all subchannels; an assumption which is hard to obtain in practice, whether the multiple-antenna configuration provides a MIMO link or is created in a distributed way.

In addressing the effect of imperfect channel knowledge in single-input single-output (SISO) and MIMO configurations, recent information-theoretical studies assume different channel state information (CSI) uncertainties at the receiver. For instance, lower and upper bounds on the capacity of SISO channels under imperfect CSI at the receiver, with and without feedback to the transmitter, are provided in [9]. In [10], the capacity in the presence of channel estimation error at the receiver is evaluated when a fixed modified nearest neighbor decoding rule is employed. The same approach has been taken in $[11,12]$ for MIMO systems with independent and identically distributed (i.i.d.) Rayleigh fading channels. In particular, it has been proven that spatio-temporal water-filling is the optimal power allocation strategy that achieves the capacity lower bound [11]. In addition, the performance of space-time coding in the presence of channel estimation error is studied in [1315]. In particular, closed-form expressions for the pairwise error probability (PEP) of space-time codes in Rayleigh flatfading channels have been obtained in [15].

In this paper, we address the effects of channel estimation error at the receiver on the performance of D-STBCs. In particular, we derive lower and upper bounds on the mutual information for Gaussian input signals, and present a limiting value that upper bounds the gap between these bounds at any input transmit powers. We further show that the gap between the mutual information bounds increases as the disparity between the subchannel estimation error variances increases. In addition, assuming that the summation of the subchannel gains remains constant, we provide the information for positioning the receiving node so as to maximize the mutual information bounds of D-STBCs. Furthermore, we provide the power allocation scheme that achieves the outage capacity lower bound of D-STBCs, and derive closed-form expressions for this capacity metric and its associated power allocation.

In detailing these contributions, the remainder of this paper is organized as follows. In Section 2, the system and channel models are introduced. Lower and upper bounds on the mutual information under channel estimation error for D-STBCs in Rayleigh fading channels are derived in Section 3. The tightness of these bounds is also analyzed in Section 3. Section 4 investigates the location of the receiver that maximizes the mutual information bounds, when the summation of the channel gains is constant. In Section 5, closed-form expressions for the lower bound on the outage capacity of D-STBCs are derived. Finally, sample numerical results are presented in Section 6 followed by the paper's conclusion.

\section{SYSTEM AND CHANNEL MODELS}

Throughout this paper, we use the upper-case boldface letters for matrices and lower-case boldface letters for vectors. $\mathbf{A}^{\mathrm{T}}, \mathbf{A}^{\mathcal{H}},|\mathbf{A}|$, and $\|\mathbf{A}\|_{\mathrm{F}}^{2}$ indicate the transpose, Hermitian transpose, determinant, and Frobenius norm of matrix A, respectively. $\mathbf{I}_{n}$ stands for an $n \times n$ identity matrix, and the matrix (pseudo) inverse is denoted by $[\cdot]^{-1} \cdot \mathcal{E}[x]$ denotes the expectation of the random variable $x$, abs $(x)$ indicates the absolute value of $x$, and $x^{*}$ its conjugate value.

We consider a wireless communication system employing $n_{\mathrm{T}}$ transmitters, each equipped with a single antenna, and a receiver equipped with $n_{\mathrm{R}}$ receive antennas in a flat-fading environment. A linear model relates the $n_{\mathrm{R}} \times 1$ received vector $\mathbf{y}$ to the signals sent from the $n_{\mathrm{T}}$ transmitting nodes, that is, $\mathbf{x}_{i}$ for $i=1, \ldots, n_{\mathrm{T}}$, via

$$
\mathbf{y}=\sum_{i=1}^{n_{\mathrm{T}}} \mathbf{h}_{i} \mathbf{x}_{i}+\mathbf{n},
$$

where the entries of $\mathbf{n}$ represent the zero-mean complex Gaussian noise with independent real and imaginary parts of equal power, and $\mathbf{h}_{i}, i=1, \ldots, n_{\mathrm{T}}$, indicate the channel transfer vector between the $i$ th transmitter and the receiver. The elements of the $n_{\mathrm{R}} \times 1$ channel transfer vectors, $\mathbf{h}_{i}$, $i=1, \ldots, n_{\mathrm{T}}$, are assumed to be independent zero-mean circularly symmetric complex Gaussian (ZMCSCG) random variables with variances $\gamma_{i}, \ldots, \gamma_{n_{\mathrm{T}}}$, referred to as channel gains.

Furthermore, we assume that the receiver performs minimum mean square error (MMSE) estimation of $\mathbf{h}_{i}, i=$ $1, \ldots, n_{\mathrm{T}}$, such that $\mathbf{h}_{i}=\hat{\boldsymbol{h}}_{i}+\mathbf{e}_{i}$, where by the property of MMSE estimation $\hat{\boldsymbol{h}}_{i}$ and $\mathbf{e}_{i}$ are uncorrelated. The elements of $\mathbf{e}_{i}, i=1, \ldots, n_{\mathrm{T}}$, are independent ZMCSCG random variables with variance $\sigma_{i}^{2}$. Finally, the average transmit power from each transmitter is constrained to $P$, and it is assumed that the transmitters cooperate to provide a distributed spacetime block encoder, and that the channel coefficients remain constant during the transmission of a space-time codeword.

\section{MUTUAL INFORMATION BOUNDS}

We start by deriving lower and upper bounds on the mutual information of the distributed system employing Alamouti codes [3], when the receiver is equipped with two antennas. Generalization to a system with $n_{\mathrm{T}}>2$ and $n_{\mathrm{R}}>2$ follows. We assume that the signals at the input of the subchannels are independent Gaussian distributed, which is not necessarily the capacity achieving distribution when CSI at the receiver is not perfect [9].

The Alamouti scheme transmits symbols $x_{1}$ and $x_{2}$ from the first and second transmitters, respectively, during the first symbol period, while symbols $-x_{2}^{*}$ and $x_{1}^{*}$ are transmitted from the first and second transmitters during the second 
symbol period, respectively. The channels between the distributed transmitters and the receiver remain unchanged during these two symbol periods. Let us define vectors $\mathbf{y}_{1}$ and $y_{2}$ as the received vectors at the first and second time periods. The receiver forms a rearranged signal vector $\mathbf{y}$ as $\mathbf{y}=\left[\begin{array}{ll}\mathbf{y}_{1} & \mathbf{y}_{2}^{*}\end{array}\right]^{T}$ that can be expressed as

$$
\mathbf{y}=\hat{\boldsymbol{H}}_{\mathrm{eff}} \mathbf{x}+\mathbf{E}_{\mathrm{eff}} \mathbf{x}+\mathbf{n},
$$

where $\mathbf{n}=\left[\begin{array}{llll}n_{1} & n_{2} & n_{3}^{*} & n_{4}^{*}\end{array}\right]^{T}$ is the vector of noise samples, $\mathbf{x}=\left[\begin{array}{ll}x_{1} & x_{2}\end{array}\right]^{T}$, and the effective channel estimation and error matrices are given by

$$
\hat{\boldsymbol{H}}_{\mathrm{eff}}=\left(\begin{array}{cc}
\hat{h}_{11} & \hat{h}_{12} \\
\hat{h}_{21} & \hat{h}_{22} \\
\hat{h}_{12}^{*} & -\hat{h}_{11}^{*} \\
\hat{h}_{22}^{*} & -\hat{h}_{21}^{*}
\end{array}\right), \quad \mathbf{E}_{\mathrm{eff}}=\left(\begin{array}{cc}
e_{11} & e_{12} \\
e_{21} & e_{22} \\
e_{12}^{*} & -e_{11}^{*} \\
e_{22}^{*} & -e_{21}^{*}
\end{array}\right)
$$

Note that the effective channel estimation is an orthogonal matrix. Then, the receiver multiplies the received vector $y$ with the Hermitian transpose of $\hat{\boldsymbol{H}}_{\text {eff }}$ to obtain

$$
\mathbf{z}=\|\hat{\mathbf{H}}\|_{\mathrm{F}}^{2} \mathbf{I}_{2} \mathbf{x}+\hat{\boldsymbol{H}}_{\mathrm{eff}}^{\mathcal{H}} \mathbf{E}_{\mathrm{eff}} \mathbf{x}+\tilde{\boldsymbol{n}},
$$

where the vector $\tilde{\boldsymbol{n}}=\hat{\boldsymbol{H}}_{\mathrm{eff}}^{\mathcal{H}} \mathbf{n}$ is zero-mean with covariance matrix $\mathcal{E}\left[\tilde{\boldsymbol{n}} \tilde{\boldsymbol{n}}^{\mathscr{H}}\right]=\sigma_{n}^{2}\|\mathbf{H}\|_{\mathrm{F}}^{2} \mathbf{I}_{2}$.

The lower and upper bounds on the mutual information can now be derived by adopting a similar approach as used in [11] yielding

$$
\begin{aligned}
& \text { Clower } \\
& =\frac{1}{n_{\mathrm{R}}} \mathcal{E}\left[\log _{2}\left|\mathbf{I}_{n_{\mathrm{R}}}+P\left(\|\hat{\mathbf{H}}\|_{\mathrm{F}}^{2}\right)^{2}\left[\sigma_{n}^{2}\|\hat{\mathbf{H}}\|_{\mathrm{F}}^{2} \mathbf{I}_{n_{\mathrm{R}}}+\operatorname{cov}\left(\hat{\boldsymbol{H}}_{\mathrm{eff}}^{\mathcal{H}} \mathbf{E}_{\mathrm{eff}} \mathbf{x}\right)\right]^{-1}\right|\right], \\
& C_{\text {upper }} \\
& =\frac{1}{n_{\mathrm{R}}} \mathcal{E}\left[\log _{2} \mid\left[P\left(\|\hat{\mathbf{H}}\|_{\mathrm{F}}^{2}\right)^{2}+\sigma_{n}^{2}\|\hat{\mathbf{H}}\|_{\mathrm{F}}^{2} \mathbf{I}_{n_{\mathrm{R}}}+\operatorname{cov}\left(\hat{\boldsymbol{H}}_{\mathrm{eff}}^{\mathscr{H}} \mathbf{E}_{\mathrm{eff}} \mathbf{x}\right)\right]\right. \\
& \left.\times\left[\sigma_{n}^{2}\|\hat{\mathbf{H}}\|_{\mathrm{F}}^{2} \mathbf{I}_{n_{\mathrm{R}}}+\operatorname{cov}\left(\hat{\boldsymbol{H}}_{\mathrm{eff}}^{\mathcal{H}} \mathbf{E}_{\mathrm{eff}} \mathbf{x} \mid \mathbf{x}\right)\right]^{-1} \mid\right],
\end{aligned}
$$

where $\operatorname{cov}\left(\hat{\boldsymbol{H}}_{\text {eff }}^{\mathcal{H}} \mathbf{E}_{\text {eff }} \mathbf{x}\right)$ indicates the covariance matrix of the random vector $\hat{\boldsymbol{H}}_{\text {eff }}^{\mathcal{H}} \mathbf{E}_{\text {eff }} \mathbf{x}$, and $\operatorname{cov}\left(\hat{\boldsymbol{H}}_{\text {eff }}^{\mathscr{H}} \mathbf{E}_{\text {eff }} \mathbf{X} \mid \mathbf{x}\right)$ denotes the covariance matrix of the random vector $\hat{\boldsymbol{H}}_{\mathrm{eff}}^{\mathscr{H}} \mathbf{E}_{\mathrm{eff}} \mathbf{x}$ given $\mathbf{x}$. Then, inserting $\mathbf{E}_{\text {eff }}$ and $\hat{\boldsymbol{H}}_{\text {eff }}$ (3) into (5), one can derive the mutual information bounds and express them according to

$$
\begin{aligned}
C_{\text {lower }} & =\mathcal{E}\left[\log _{2}\left(1+P \frac{\|\hat{\mathbf{H}}\|_{\mathrm{F}}^{2}}{\sigma_{n}^{2}+P\left(\sigma_{1}^{2}+\sigma_{2}^{2}\right)}\right)\right], \\
C_{\text {upper }} & =\mathcal{E}\left[\log _{2}\left(\frac{P\|\hat{\mathbf{H}}\|_{\mathrm{F}}^{2}+\sigma_{n}^{2}+P\left(\sigma_{1}^{2}+\sigma_{2}^{2}\right)}{\sigma_{n}^{2}+P\left(\sigma_{1}^{2} \chi_{1}^{2}+\sigma_{2}^{2} \chi_{2}^{2}\right)}\right)\right],
\end{aligned}
$$

where $\mathcal{X}_{i}^{2}, i \in\{1,2\}$, is a chi-squared random variable with two degrees of freedom and $\mathcal{E}\left[\mathcal{X}_{i}^{2}\right]=1$. Note that the term $P\left(\sigma_{1}^{2}+\sigma_{2}^{2}\right)$, appearing in the mutual information lower bound
(6), can be seen as the variance of an additive white Gaussian noise (AWGN).

Furthermore, by following similar steps as in (2) to (7), one can find the mutual information lower and upper bounds of D-STBCs with arbitrary numbers of transmit and receive antennas such that

$$
\begin{aligned}
& C_{\text {lower }}=R \mathcal{E}\left[\log _{2}\left(1+\frac{1}{R} \frac{P\|\hat{\mathbf{H}}\|_{\mathrm{F}}^{2}}{\sigma_{n}^{2}+P \sum_{i=1}^{n_{\mathrm{T}}} \sigma_{i}^{2}}\right)\right], \\
& C_{\text {upper }}=R \mathcal{E}\left[\log _{2}\left(\frac{1}{R} \frac{P\|\hat{\mathbf{H}}\|_{\mathrm{F}}^{2}+R\left(\sigma_{n}^{2}+P \sum_{i=1}^{n_{\mathrm{T}}} \sigma_{i}^{2}\right)}{\sigma_{n}^{2}+P \sum_{i=1}^{n_{\mathrm{T}}} \sigma_{i}^{2} X_{i}^{2}}\right)\right],
\end{aligned}
$$

where $R$ denotes the communication rate of the STBC.

We now investigate the tightness of the obtained lower and upper bounds on the mutual information to justify that they represent a good estimate of the true Gaussian mutual information. Define $\Delta$ as the gap between the mutual information bounds:

$$
\Delta=R \mathcal{E}\left[\log _{2}\left(\frac{\sigma_{n}^{2}+P \sum_{i=1}^{n_{\mathrm{T}}} \sigma_{i}^{2}}{\sigma_{n}^{2}+P \sum_{i=1}^{n_{\mathrm{T}}} \sigma_{i}^{2} \chi_{i}^{2}}\right)\right]
$$

then an upper bound on $\Delta$ at high transmit powers can be derived by adopting similar approach to that in [16] as follows:

$$
\lim _{\substack{P \rightarrow \infty \\ n_{\mathrm{T}} \rightarrow \infty}} \Delta \leq R \cdot \min \left\{\frac{\varepsilon}{\ln 2}, \frac{1}{2 n_{\mathrm{T}} \ln 2}+\log _{2}\left(\frac{\sigma_{\max }^{2}}{\sigma_{\min }^{2}}\right)\right\},
$$

where $\sigma_{\min }^{2}$ and $\sigma_{\max }^{2}$ are the minimum and maximum values of $\sigma_{i}^{2}$ for $i=1, \ldots, n_{\mathrm{T}}$, respectively, and $\varepsilon=0.577216$ is the Euler-Mascheroni constant [17]. Furthermore, the gap between the mutual information bounds is shown to increase monotonically as a function of the input transmit power [18]; hence, $\Delta$ does not exceed the right-hand side of (10), or equivalently, the mutual information bounds are fairly close at any input transmit powers.

We now assume that the receiver can estimate the channels pertaining to different transmitters with the same accuracy, that is, $\sigma_{1}^{2}=\cdots=\sigma_{n_{\mathrm{T}}}^{2} \triangleq \sigma_{e}^{2}$. In this case, the gap between the mutual information bounds can be shown to be upper bounded by $\lim _{P \rightarrow \infty, n_{\mathrm{T}} \rightarrow \infty} \Delta \leq R /\left(2 n_{\mathrm{T}} \ln 2\right)$, which shows that the gap between the mutual information bounds decreases as the number of transmitters increases.

Proceeding with our investigation about the gap between the mutual information bounds, we now provide the following lemma.

Lemma 1. The gap between the bounds on the mutual information of distributed Alamouti codes with unequal channel error variances increases monotonically as the disparity between the error variances increases.

Proof. Consider that the channel error variances $\sigma_{1}^{2}$ and $\sigma_{2}^{2}$ are respectively given by $\sigma_{\text {sum }}^{2}-\alpha_{e}$ and $\sigma_{\text {sum }}^{2}+\alpha_{e}$. The 
gap between the mutual information bounds, $\Delta$, can be simplified to

$$
\Delta=R \mathcal{E}\left[\log _{2}\left(\frac{\sigma_{n}^{2}+P \sigma_{\text {sum }}^{2}}{\sigma_{n}^{2}+P\left(\left(\sigma_{\text {sum }}^{2}-\alpha_{e}\right) \mathcal{X}_{1}^{2}+\left(\sigma_{\text {sum }}^{2}+\alpha_{e}\right) X_{2}^{2}\right)}\right)\right] .
$$

We now find the first partial derivative of $\Delta$ with respect to $\alpha_{e}$ and prove that $\Delta$ is an increasing function of $\alpha_{e}$. We proceed as follows:

$$
\frac{\partial \Delta}{\partial \alpha_{e}}=\frac{R}{\ln 2} \varepsilon\left[\frac{P\left(X_{1}^{2}-X_{2}^{2}\right)}{\sigma_{n}^{2}+P\left(\left(\sigma_{\text {sum }}^{2}-\alpha_{e}\right) X_{1}^{2}+\left(\sigma_{\text {sum }}^{2}+\alpha_{e}\right) X_{2}^{2}\right)}\right] .
$$

Then, by using the fact that $\mathcal{X}_{1}^{2}$ and $X_{2}^{2}$ are i.i.d. random variables, we can show that $\partial \Delta /\left.\partial \alpha_{e}\right|_{\alpha_{e}=0}=0$. Furthermore, one can now derive the second partial derivative of $\Delta$ with respect to $\alpha_{e}$ which leads to $\partial^{2} \Delta / \partial \alpha_{e}^{2} \geq 0$. This implies that $\partial \Delta / \partial \alpha_{e}$ is an increasing function of $\alpha_{e}$, hence, $\partial \Delta / \partial \alpha_{e} \geq 0$ for $0 \leq \alpha_{e} \leq 1$, which concludes the proof.

\section{OPTIMUM POSITIONING}

In the communication system under consideration, we now assume that the transmitters are fixed in their position and that the receiver can estimate the channel gains pertaining to different transmitters with the same accuracy, and investigate the best position for the receiving node. Our transmitters can be sensor nodes placed, for example, at the corners of a room, and we investigate the best location of the receiver collecting data from these nodes, where we assume that nodes cooperate to provide a distributed space-time block coded transmission. In particular, we assume that when the channel gains pertaining to a subset of transmitter-receiver links improve, the gains of the rest of the subchannels degrade such that the summation of all gains remains constant, and provide the following lemma.

Lemma 2. The mutual information bounds of D-STBCs are maximum when the channel gains pertaining to different transmitter-receiver links are equal, as long as the summation of these gains remains constant.

Proof. The proof for this lemma can be obtained by adopting a similar approach as proposed in [19]. For completeness, we provide here the proof for a system with $n_{\mathrm{T}}=3$ transmitting nodes and a single receive antenna.

We refer to the channel gains by $\gamma_{1}, \gamma_{2}$, and $\gamma_{3}$, and define the constant $3 \beta$ as the summation of these variances, that is, $\sum_{i=1}^{3} \gamma_{i}=3 \beta$. Since the channel gains are real positive numbers, then at least one of them is bigger than or equal to $\beta$. Without loss of generality, we assume that $\gamma_{1} \geq \beta$ and define $0 \leq \alpha_{1} \leq 1$ such that $\gamma_{1}=\beta\left(1+2 \alpha_{1}\right)$. Hence, summation of the two remaining channel gains, $\gamma_{2}$ and $\gamma_{3}$, can be found as $\gamma_{2}+\gamma_{3}=2 \beta\left(1-\alpha_{1}\right)$. Furthermore, we define $0 \leq \alpha_{2} \leq 1$ such that $\gamma_{2}=\beta\left(1-\alpha_{1}\right)\left(1+\alpha_{2}\right)$ and $\gamma_{3}=\beta\left(1-\alpha_{1}\right)\left(1-\alpha_{2}\right)$. We can then simplify the mutual information lower bound (8) as follows:

$$
\begin{aligned}
C_{\text {lower }} & =R \mathscr{E}\left[\log _{2}\left(1+\frac{P}{R} \frac{\gamma_{1} w_{1}+\gamma_{2} w_{2}+\gamma_{3} w_{3}-\sigma_{e}^{2} \sum_{i=1}^{3} w_{i}}{\sigma_{n}^{2}+3 P \sigma_{e}^{2}}\right)\right] \\
& =R \&\left[\log _{2}(1+Q)\right],
\end{aligned}
$$

where $Q=a\left(\left(1+2 \alpha_{1}\right) w_{1}+\left(1-\alpha_{1}\right)\left(1+\alpha_{2}\right) w_{2}+\left(1-\alpha_{1}\right)(1-\right.$ $\left.\left.\alpha_{2}\right) w_{3}-\sigma_{e}^{2} / \beta \sum_{i=1}^{3} w_{i}\right), \sigma_{e}^{2}$ represents the channel estimation error variance, $w_{i}, i=1, \ldots, 3$, are i.i.d. random variables according to Rayleigh distribution with unit variances, and $a=P \beta /\left(R\left(\sigma_{n}^{2}+3 P \sigma_{e}^{2}\right)\right)$. We need to prove that $C_{\text {lower }}$ is at its maximum when $\alpha_{1}=0$ and $\alpha_{2}=0$. We start by deriving the first and second partial derivatives of $C_{\text {lower }}$ with respect to $\alpha_{2}$ :

$$
\begin{gathered}
\frac{\partial C_{\text {lower }}}{\partial \alpha_{2}}=\frac{R}{\ln 2} \varepsilon\left[\frac{a\left(1-\alpha_{1}\right)\left(w_{2}-w_{3}\right)}{1+Q}\right], \\
\frac{\partial^{2} C_{\text {lower }}}{\partial \alpha_{2}^{2}}=-\frac{R}{\ln 2} \varepsilon\left[\frac{a\left(1-\alpha_{1}\right)\left(w_{2}-w_{3}\right)}{1+Q}\right]^{2} .
\end{gathered}
$$

Observe that the second derivative of $C_{\text {lower }}$ with respect to $\alpha_{2}$ (15) is nonpositive, therefore, the maximum on $\partial C_{\text {lower }} / \partial \alpha_{2}$ (14) occurs at $\alpha_{2}=0$, irrespective of $\alpha_{1}$. Furthermore, by adopting similar steps as in [16], one can show that $\partial C_{\text {lower }} /\left.\partial \alpha_{2}\right|_{\alpha_{2}=0}=0$, which proves that the maximum on the mutual information lower bound occurs at $\alpha_{2}=0$ for any value of $\alpha_{1}$. Note that since abs $\left(\partial C_{\text {lower }} / \partial \alpha_{2}\right)$ increases monotonically as a function of $\alpha_{2}$, then not only the mutual information lower bound is at its maximum when $\alpha_{2}=0$, but, its robustness to the variations of $\alpha_{2}$ is also maximum at this point.

We now prove that the maximum of $\left.C_{\text {lower }}\right|_{\alpha_{2}=0}$ occurs at $\alpha_{1}=0$. For this purpose, we define the function $f\left(\alpha_{1}\right)=$ $\left.C_{\text {lower }}\right|_{\alpha_{2}=0}$ given by

$$
f\left(\alpha_{1}\right)=R \mathscr{E}\left[\log _{2}\left(1+Q^{\prime}\right)\right]
$$

where $Q^{\prime}=a\left(\left(1+2 \alpha_{1}\right) w_{1}+\left(1-\alpha_{1}\right) w_{2}+\left(1-\alpha_{1}\right) w_{3}-\right.$ $\left.\sigma_{e}^{2} / \beta \sum_{i=1}^{3} w_{i}\right)$. Then, by obtaining the first and second derivatives of $f\left(\alpha_{1}\right)$ with respect to $\alpha_{1}$, one can show that $\partial^{2} f\left(\alpha_{1}\right) / \partial \alpha_{1}^{2} \leq 0$ and $\partial f\left(\alpha_{1}\right) /\left.\partial \alpha_{1}\right|_{\alpha_{1}=0}=0$, hence, the maximum of $f\left(\alpha_{1}\right)$ occurs at $\alpha_{1}=0$. Therefore, the maximum of $C_{\text {lower }}$ occurs at $\alpha_{1}=0$ and $\alpha_{2}=0$.

In addition, since the gap between the mutual information bounds, $\Delta$, does not depend on the variations of channel gains, then the mutual information upper bound is also maximum at $\alpha_{1}=0$ and $\alpha_{2}=0$; which concludes the proof.

According to the above analysis, one can conclude that the best position for the receiving node is the one that provides the condition of having equal subchannel gains. For instance, when the distributed transmit antennas are located 
in the corners of a room, the best position for the receiving node is the center of the room, under the condition that the summation of the subchannel gains remains constant.

\section{OUTAGE CAPACITY}

In the following, we assume that the transmitters, considered to cooperate to provide a distributed STBCed transmission, can adaptively change their input power according to the channel variations. The transmitting nodes use the same input power level, which can be calculated at the receiver that has access to the state information of each subchannel. The receiver then broadcasts the information about the required transmit power level, and the transmitters adapt their input power according to this information. Here, we investigate the adaptive power allocation scheme that achieves the outage capacity lower bound of the channel.

Outage capacity is the maximum constant-rate that can be achieved with an outage probability less than a certain threshold [20, 21]. In this case, the transmitters invert the channel fading so as to maintain a constant power at the receiver. Using channel inversion, the capacity of fading channels and its closed-form expressions have previously been derived in $[22,23]$, respectively. This metric corresponds to the capacity that can be achieved in all fading states while meeting the power constraint. However, in extreme fading cases, for example, Rayleigh fading, this capacity is zero as the transmitter has to spend a huge amount of power for channel states in deep fade to achieve a constant rate. To alleviate this problem, an adaptive transmission technique, referred to as truncated channel inversion with fixed rate (tifr), which can achieve nonzero constant rates, was introduced in [22]. This technique maintains a constant received-power for channel fades above a given cutoff depth.

Recalling that channel inversion technique provides a constant received power at the receiver such that $(1 / R)\left(P\|\hat{\mathbf{H}}\|_{\mathrm{F}}^{2} /\left(\sigma_{n}^{2}+P \sum_{i=1}^{n_{\mathrm{T}}} \sigma_{i}^{2}\right)\right)=\alpha$, we can find the power allocation for the system with D-STBCs and imperfect channel estimation at the receiver according to

$$
P=\left[\frac{\alpha R \sigma_{n}^{2}}{\|\hat{\mathbf{H}}\|_{\mathrm{F}}^{2}-\alpha R \sum_{i=1}^{n_{\mathrm{T}}} \sigma_{i}^{2}}\right]^{+},
$$

where the constant value for $\alpha$ is found such that the transmit-power constraint is satisfied and $[x]^{+}$denotes $\max \{0, x\}$. We assume that the transmission is suspended for channel gains below a cutoff threshold $\lambda_{0}$ such that the outage probability $P_{\text {out }}$ is satisfied. Note that, at the same time, the transmission is suspended for channel gains smaller than $\|\hat{\boldsymbol{H}}\|_{\mathrm{F}}^{2} \leq \alpha R \sum_{i=1}^{n_{\mathrm{T}}} \sigma_{i}^{2}$; hence, the acceptable value for $\alpha$ is limited to $\alpha \leq \lambda_{0} /\left(R \sum_{i=1}^{n_{\mathrm{T}}} \sigma_{i}^{2}\right)$. Therefore, the lower bound on the outage capacity can be obtained as

$$
C_{\text {out }}=R \log _{2}\left(1+\min \left(\alpha, \frac{\lambda_{0}}{R \sum_{i=1}^{n_{\mathrm{T}}} \sigma_{i}^{2}}\right)\right) \operatorname{Pr}\left\{\|\hat{\mathbf{H}}\|_{\mathrm{F}}^{2} \geq \lambda_{0}\right\},
$$

where $\operatorname{Pr}\left\{\|\hat{\boldsymbol{H}}\|_{\mathrm{F}}^{2} \geq \lambda_{0}\right\}=1-P_{\text {out }}$ indicates the probability that the inequality $\|\hat{\boldsymbol{H}}\|_{\mathrm{F}}^{2} \geq \lambda_{0}$ holds true. It is worth noting that the expression derived in (18) does not represent the true channel outage capacity. However, one can guarantee that by using the power allocation scheme in (17), at least a minimum constant-rate according to (18) can be achieved by D-STBCs with imperfect CSI at the receiver. Also, recalling that the mutual information bounds (8) are proved to be tight at any input transmit powers, we conclude that (18) represents a good estimate for the true channel outage capacity. Hereafter, we use the parameter $\lambda=\|\hat{\boldsymbol{H}}\|_{\mathrm{F}}^{2}$ for the ease of notation.

To obtain a closed-form expression for the outage capacity, we start by deriving a closed-form expression for $\operatorname{Pr}\left\{\lambda \geq \lambda_{0}\right\}$. We proceed by defining $u_{i}$ as the number of transmitters with equal $\gamma_{i}-\sigma_{i}^{2}$ and choose $g$ such that $\sum_{i=1}^{g} u_{i}=n_{\mathrm{T}}$. Without loss of generality, we assume that $\gamma_{l}-\sigma_{l}^{2} \neq \gamma_{k}-\sigma_{k}^{2}$ for $l=1, \ldots, g$ and $k=1, \ldots, g$, having $k \neq l$. The probability density function (PDF) of $\lambda, f_{\lambda}(\lambda)$, can now be found by following similar steps as in [7] according to

$$
f_{\lambda}(\lambda)=\sum_{i=1}^{g} \sum_{j=1}^{u_{i}} K_{i, j} \frac{\lambda^{j-1}}{\Gamma(j)\left(\gamma_{i}-\sigma_{i}^{2}\right)^{j}} e^{-\lambda /\left(\gamma_{i}-\sigma_{i}^{2}\right)},
$$

where $\Gamma(\cdot)$ is the Gamma function [24], and the coefficients $K_{i, j}$ are given by

$$
\begin{aligned}
K_{i, j}= & \frac{1}{\left(u_{i}-j\right) !\left(-\gamma_{i}+\sigma_{i}^{2}\right)^{u_{i}-j}} \\
& \times \frac{\partial^{u_{i}-j}}{\partial s^{u_{i}-j}}\left[\prod_{k=1, k \neq i}^{g}\left(1-\left(\gamma_{k}-\sigma_{k}^{2}\right) s\right)^{-u_{k}}\right]_{s=1 /\left(\gamma_{i}-\sigma_{i}^{2}\right)}
\end{aligned}
$$

We can then obtain a closed-form solution for the probability $\operatorname{Pr}\left\{\lambda \geq \lambda_{0}\right\}=\int_{\lambda_{0}}^{\infty} f_{\lambda}(\lambda) d \lambda$ as follows:

$$
\operatorname{Pr}\left\{\lambda \geq \lambda_{0}\right\}=\sum_{i=1}^{g} \sum_{j=1}^{u_{i}} \sum_{k=0}^{j-1} \frac{K_{i, j} e^{-\lambda_{0} /\left(\gamma_{i}-\sigma_{i}^{2}\right)} \lambda_{0}^{k}}{\left(\gamma_{i}-\sigma_{i}^{2}\right)^{k} \Gamma(k+1)}
$$

On the other hand, given that the transmission is suspended for the channel gains below the cutoff threshold, $\lambda_{0}$, we can find a closed-form expression for $\alpha$ by expanding the input power constraint as

$$
\begin{aligned}
P & =\int_{\lambda_{0}}^{\infty} \frac{\alpha R \sigma_{n}^{2}}{\lambda-\alpha R \sum_{i=1}^{n_{\mathrm{T}}} \sigma_{i}^{2}} f_{\lambda}(\lambda) d \lambda \\
& =\sum_{i=1}^{g} \sum_{j=1}^{u_{i}} \frac{\alpha R \sigma_{n}^{2} K_{i, j}}{\Gamma(j) m_{i}^{j}} \int_{\lambda_{0}}^{\infty} \frac{\lambda^{j-1}}{(\lambda-n)} e^{-\lambda / m_{i}} d \lambda,
\end{aligned}
$$


where $m_{i}=\gamma_{i}-\sigma_{i}^{2}$ and $n=\alpha R \sum_{i=1}^{n_{\mathrm{T}}} \sigma_{i}^{2}$. The integration in (22) can be expanded by using the equalities $\lambda^{j-1}-n^{j-1}=$ $(\lambda-n) \sum_{k=0}^{j-2} n^{k} \lambda^{j-2-k}, \int x^{j} e^{-x / m_{i}} d x=-m_{i} e^{-x / m_{i}} \sum_{l=0}^{j}(j ! /(j-$ l)!) $m_{i}^{l} x^{j-l}$, and $\int\left(n^{j-1} /(x-n)\right) e^{-x / m_{i}} d x=n^{j-1} e^{-n / m_{i}} \operatorname{Ei}((n-$ $x) / m_{i}$ ) such that

$$
\begin{aligned}
P= & \alpha \sum_{i=1}^{g} \sum_{j=1}^{u_{i}} \frac{R \sigma_{n}^{2} K_{i, j}}{\Gamma(j) m_{i}^{j}} \\
& \times\left(\sum_{k=0}^{j-2} m_{i} n^{k} e^{-\lambda_{0} / m_{i}} \sum_{l=0}^{j-2-k} \frac{\Gamma(j-k-1)}{\Gamma(j-k-l-1)} m_{i}^{l} \lambda_{0}^{j-k-l-2}\right. \\
& \left.\quad-n^{j-1} e^{-n / m_{i}} \operatorname{Ei}\left(\frac{n-\lambda_{0}}{m_{i}}\right)\right)
\end{aligned}
$$

which leads to a closed-form expression for $\alpha$.

\section{NUMERICAL RESULTS}

In this section, we provide some numerical results in order to illustrate our theoretical analysis. For our simulations, we consider distributed Alamouti codes in Rayleigh fading channels and assume that $\mathrm{SNR}=P / \sigma_{n}^{2}$ and $\sigma_{n}^{2}=1$; hence, a high SNR implies a high transmit power in the presented results.

We start by comparing the mutual information bounds, $C_{\text {lower }}$ and $C_{\text {upper }}$, of D-STBCs with the same subchannel estimation error variances, $\sigma_{1}^{2}=\sigma_{2}^{2}=\sigma_{e}^{2}$, and with a single receive antenna for different values of $\sigma_{e}^{2}$. In Figure 1, the channel gains from the two transmitters are assumed to be $\gamma_{1}=1.5$ and $\gamma_{2}=0.5$. The steady and dashed lines correspond to the mutual information lower and upper bounds, respectively. Figure 1 shows that not only are the bounds fairly close at high SNRs, but also that the gap between the two bounds is small for low SNRs. We observe that at low SNRs, the capacity increases logarithmically as a function of SNR, but with smaller slope as compared to a system with perfect CSI at the receiver, that is, when $\sigma_{e}^{2}=0$. Figure 1 also indicates that at high SNRs, the mutual information bounds saturate and do not increase logarithmically as a function of SNR.

The gap between the mutual information bounds, $\Delta$, for D-STBCs with two receive antennas and a constant measure for $\sigma_{1}^{2}+\sigma_{2}^{2}$, namely, $\sigma_{1}^{2}+\sigma_{2}^{2}=0.2$, are plotted versus SNR in Figure 2. The plots show that when the SNR increases, $\Delta$ increases monotonically. The figure also illustrates that the gap between the mutual information bounds increases when the ratio between the subchannel estimation error variances, that is, $\sigma_{1}^{2} / \sigma_{2}^{2}$, increases.

In Figure 3, we further plot the gap between the mutual information bounds for D-STBCs, SIMO subchannels, and distributed MIMO channel with two receive antennas, versus the channel estimation error variance of the first subchannel, that is, $\sigma_{1}^{2}$, at $\mathrm{SNR}=20 \mathrm{~dB}$. The channel estimation error variance of the second subchannel relates to $\sigma_{1}^{2}$ through $\sigma_{1}^{2}+$ $\sigma_{2}^{2}=0.1$. The figure shows that the gap between the mutual information bounds of D-STBCs is relatively small compared

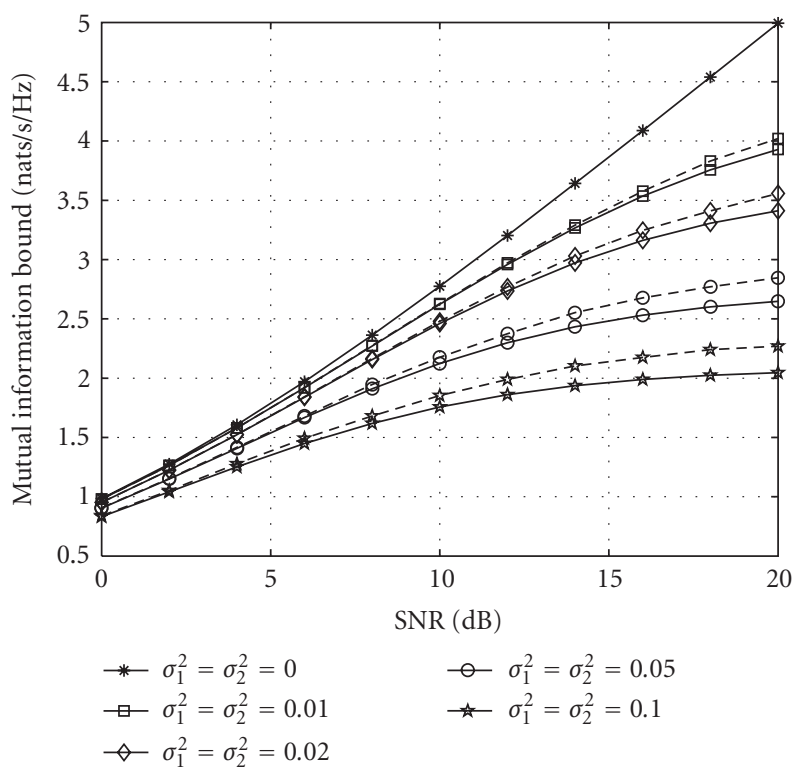

Figure 1: Mutual information lower and upper bounds for DSTBCs with single receive antenna; $\sigma_{1}^{2}=\sigma_{2}^{2}$.

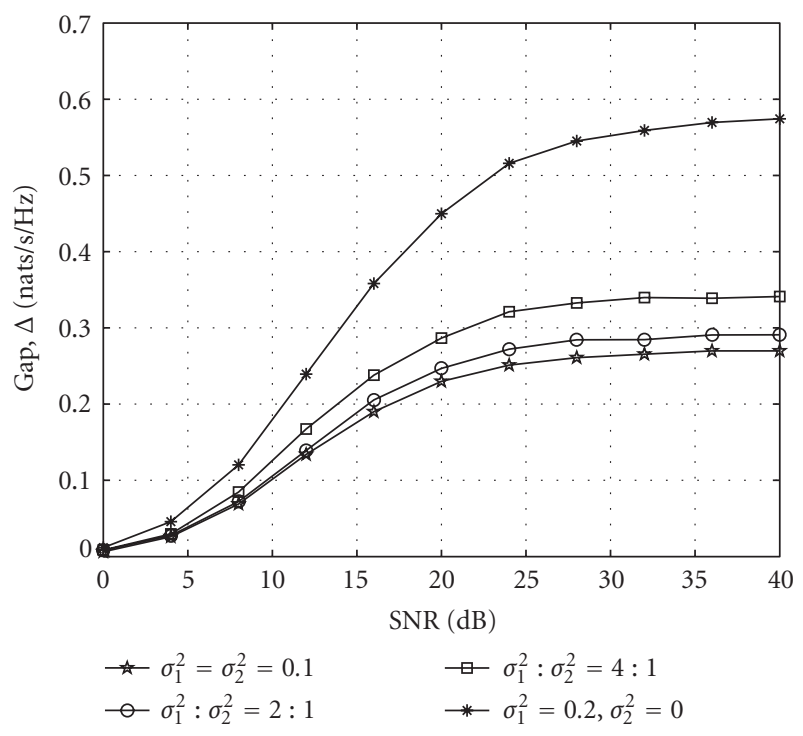

FIgURe 2: Gap between the mutual information bounds for DSTBCs with two receive antennas; $\sigma_{1}^{2}+\sigma_{2}^{2}=0.2$.

to that of the SIMO and distributed MIMO channels. We also observe that the gap for D-STBCs changes slowly as the subchannel estimation error variances change, while $\Delta$ in SIMO subchannels increases significantly when the channel estimation error variance increases.

The mutual information lower bound of D-STBCs with a single receive antenna and with $\gamma_{1}=1+\alpha_{\gamma}$ and $\gamma_{2}=$ $1-\alpha_{y}$ is plotted in Figure 4 for SNR $=15 \mathrm{~dB}$. Variations of the bounds as a function of $\alpha_{\gamma}$ are illustrated for various channel estimation error variances showing that the mutual information lower bound is at its maximum when $\alpha_{\gamma}=0$, or equivalently, when $\gamma_{1}=\gamma_{2}$; hence confirming the results of 


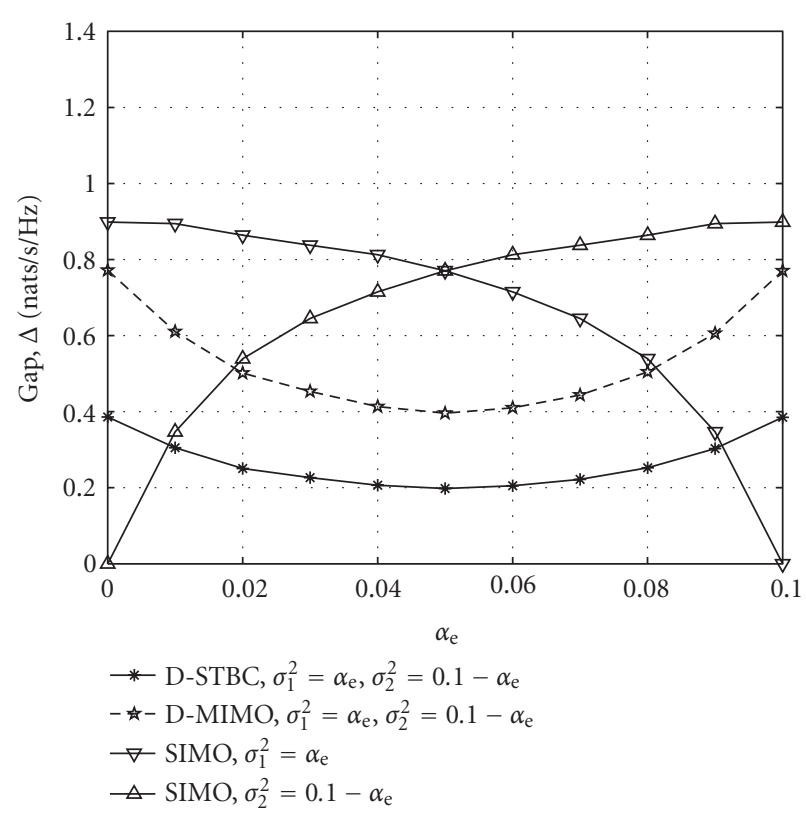

Figure 3: Gap between the mutual information bounds for DSTBCs with two receive antennas, and for its SIMO subchannels at $\mathrm{SNR}=20 \mathrm{~dB}$ : variations as a function of $\sigma_{1}^{2}=\alpha_{e}$ given $\sigma_{1}^{2}+\sigma_{2}^{2}=0.1$.

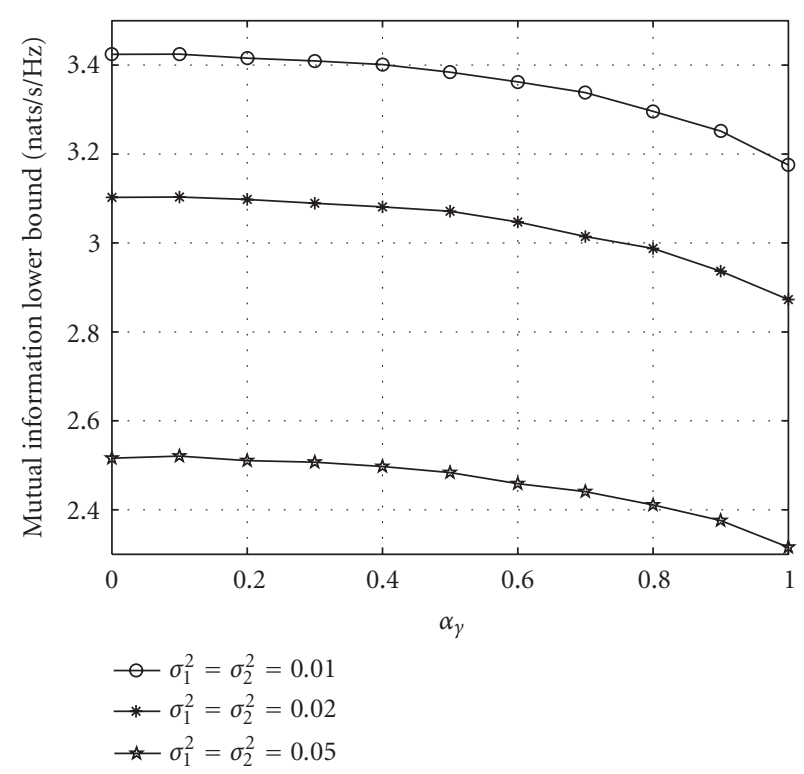

FIGURE 4: Mutual information lower bounds for D-STBCs with single receive antenna at $\mathrm{SNR}=15 \mathrm{~dB}$, given $\gamma_{1}=1+\alpha_{\gamma}$ and $\gamma_{2}=1-\alpha_{\gamma}$.

Section 4. The figure also illustrates that the variations of the mutual information lower bound as a function of $\alpha_{\gamma}$ is small around $\alpha_{\gamma}=0$.

In Figure 5, the outage capacity lower bound of D-STBCs with $\gamma_{1}=1.5$ and $\gamma_{2}=0.5$ and with a single receive antenna is plotted versus SNR for different values of $P_{\text {out }}$. The plots show that the outage capacity suffers a significant loss as a result of estimation errors at the receiver. Indeed, it can be

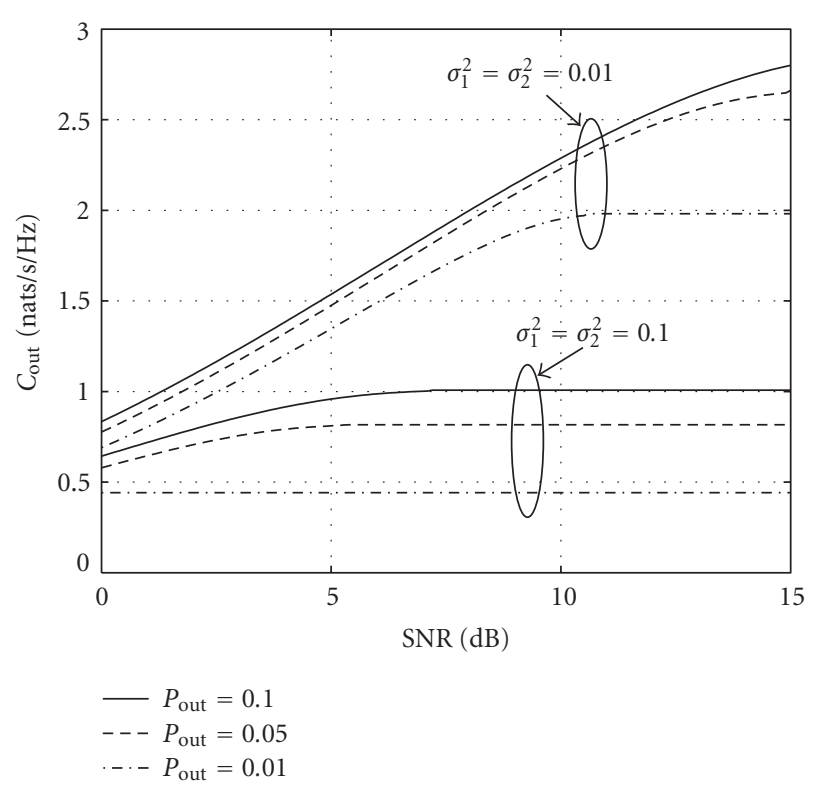

FIGURE 5: Lower bounds on the outage capacity of D-STBCs with single receive antenna.

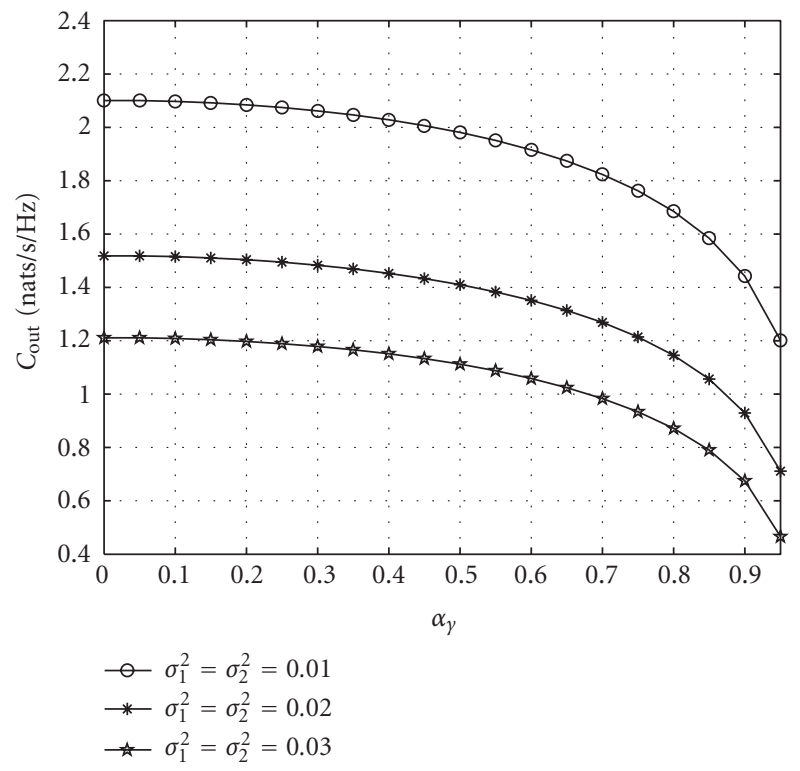

FIGURE 6: Lower bound on the outage capacity of D-STBCs with single receive antenna versus the channel gains variations at $\mathrm{SNR}=$ $15 \mathrm{~dB}$.

seen that the outage capacity of D-STBCs with $\sigma_{1}^{2}=\sigma_{2}^{2}=0.1$ starts to saturate at SNR values as small as $5 \mathrm{~dB}$.

Finally in Figure 6, the lower bound on the outage capacity of D-STBCs with outage probability $P_{\text {out }}=1 \%$ and with subchannel gains $\gamma_{1}=1+\alpha_{\gamma}$ and $\gamma_{2}=1-\alpha_{\gamma}$ is plotted versus $\alpha_{\gamma}$ at $\mathrm{SNR}=15 \mathrm{~dB}$ for various channel estimation error variances. The figure shows that a capacity gain of 0.9 nats/s/Hz can be achieved by positioning the receiver such 
that it provides $\gamma_{1}=\gamma_{2}$. Furthermore, comparing Figures 4 and 6 reveals that by optimum positioning, the increase in the capacity of a system with channel inversion technique is higher than that of a system with constant input power transmission.

\section{CONCLUSION}

We have addressed the effect of channel knowledge uncertainty at the receiver on the mutual information of distributed space-time block coded transmission in Rayleigh fading channels. Specifically, we studied upper and lower bounds on the mutual information of the system when knowledge of the variance of the channel estimation error is available at the receiver and the transmitters. We provided a limiting value that upper bounds the gap between the mutual information bounds at any input transmit powers so as to justify that they represent a good estimate of the true channel mutual information for Gaussian input signals. We also showed that the tightness between the bounds increases when the number of transmitters increases as long as the receiver can estimate the channels pertaining to different transmitters with the same accuracy. In addition, we showed that when the disparity between the estimation error variances increases, the gap between the bounds increases. Also, assuming that the summation of the channel gains is constant, we determined the receiver's position at which the mutual information lower and upper bounds of D-STBCs and their robustness to the variations of the subchannel gains are maximum. We further determined a lower bound for the outage capacity of D-STBCs with arbitrary numbers of transmit and receive antennas, and also obtained closedform expressions for this capacity metric and its associated power allocation scheme. Numerical results showed that the capacity increase, achieved by optimum positioning of the receiver, is higher in systems with channel inversion transmission technique as compared to constant input power transmission, and that the outage capacity suffers significant loss as a result of channel estimation errors at the receiver.

\section{ACKNOWLEDGMENTS}

This work was supported in part by the Natural Sciences and Engineering Research Council (NSERC) of Canada. Part of this work was presented at IEEE WCNC'07.

\section{REFERENCES}

[1] V. Tarokh, N. Seshadri, and A. R. Calderbank, "Space-time codes for high data rate wireless communication: performance criterion and code construction," IEEE Transactions on Information Theory, vol. 44, no. 2, pp. 744-765, 1998.

[2] V. Tarokh, H. Jafarkhani, and A. R. Calderbank, "Space-time block codes from orthogonal designs," IEEE Transactions on Information Theory, vol. 45, no. 5, pp. 1456-1467, 1999.

[3] S. M. Alamouti, "A simple transmit diversity technique for wireless communications," IEEE Journal on Selected Areas in Communications, vol. 16, no. 8, pp. 1451-1458, 1998.
[4] E. G. Larsson and P. Stoica, Space-Time Block Coding for Wireless Communications, Cambridge University Press, New York, NY, USA, 2003.

[5] A. Maaref and S. Aïssa, "Performance analysis of orthogonal space-time block codes in spatially correlated MIMO Nakagami fading channels," IEEE Transactions on Wireless Communications, vol. 5, no. 4, pp. 807-817, 2006.

[6] J. N. Laneman and G. W. Wornell, "Distributed space-timecoded protocols for exploiting cooperative diversity in wireless networks," IEEE Transactions on Information Theory, vol. 49, no. 10, pp. 2415-2425, 2003.

[7] M. Dohler and H. Aghvami, "Information outage probability of distributed STBCs over Nakagami fading channels," IEEE Communications Letters, vol. 8, no. 7, pp. 437-439, 2004.

[8] A. Hjørungnes and D. Gesbert, "Precoding of orthogonal space-time block codes in arbitrarily correlated MIMO channels: iterative and closed-form solutions," IEEE Transactions on Wireless Communications, vol. 6, no. 3, pp. 1072-1082, 2007.

[9] M. Médard, "The effect upon channel capacity in wireless communications of perfect and imperfect knowledge of the channel," IEEE Transactions on Information Theory, vol. 46, no. 3, pp. 933-946, 2000.

[10] A. Lapidoth and S. Shamai, "Fading channels: how perfect need "perfect side information" be?" IEEE Transactions on Information Theory, vol. 48, no. 5, pp. 1118-1134, 2002.

[11] T. Yoo and A. Goldsmith, "Capacity and power allocation for fading MIMO channels with channel estimation error," IEEE Transactions on Information Theory, vol. 52, no. 5, pp. 22032214, 2006.

[12] A. Sabharwal, E. Erikp, and B. Aazhang, "On channel state information in multiple antenna block fading channels," in Proceedings of International Symposium on Information Theory and Its Applications, pp. 116-119, Honolulu, Hawaii, USA, November 2000.

[13] V. Tarokh, A. Naguib, N. Seshadri, and A. R. Calderbank, "Space-time codes for high data rate wireless communication: performance criteria in the presence of channel estimation errors, mobility, and multiple paths," IEEE Transactions on Communications, vol. 47, no. 2, pp. 199-207, 1999.

[14] W. Hoteit, Y. R. Shayan, and A. K. Elhakeem, "Effects of imperfect channel estimation on space-time coding performance," IEE Proceedings-Communications, vol. 152, no. 3, pp. 277-281, 2005.

[15] P. Garg, R. K. Mallik, and H. M. Gupta, "Performance analysis of space-time coding with imperfect channel estimation," IEEE Transactions on Wireless Communications, vol. 4, no. 1, pp. 257-265, 2005.

[16] L. Musavian, M. R. Nakhai, M. Dohler, and A. H. Aghvami, "Effect of channel uncertainty on the mutual information of MIMO fading channels," IEEE Transactions on Vehicular Technology, vol. 56, no. 5, part 1, pp. 2798-2806, 2007.

[17] R. M. Young, "Euler's constant," The Mathematical Gazette, vol. 75, no. 472, pp. 187-190, 1991.

[18] L. Musavian and S. Aïssa, "Performance analysis of distributed space-time coded transmission with channel estimation error," in Proceedings of the IEEE Wireless Communications and Networking Conference (WCNC '07), pp. 1275-1280, Hong Kong, China, March 2007.

[19] L. Musavian and S. Aïssa, "On the achievable sum-rate of correlated MIMO multiple access channel with imperfect channel estimation," to appear in IEEE Transactions on Wireless Communications. 
[20] E. Biglieri, J. Proakis, and S. Shamai, "Fading channels: information-theoretic and communications aspects," IEEE Transactions on Information Theory, vol. 44, no. 6, pp. 26192692, 1998.

[21] G. Caire and S. Shamai, "On the capacity of some channels with channel state information," IEEE Transactions on Information Theory, vol. 45, no. 6, pp. 2007-2019, 1999.

[22] A. J. Goldsmith and P. P. Varaiya, "Capacity of fading channels with channel side information," IEEE Transactions on Information Theory, vol. 43, no. 6, pp. 1986-1992, 1997.

[23] M.-S. Alouini and A. J. Goldsmith, "Capacity of Rayleigh fading channels under different adaptive transmission and diversity-combining techniques," IEEE Transactions on Vehicular Technology, vol. 48, no. 4, pp. 1165-1181, 1999.

[24] M. Abramowitz and I. A. Stegun, Handbook of Mathematical Functions, with Formulas, Graphs, and Mathematical Tables, Dover, New York, NY, USA, 1965. 